\section{Josée Pagnier Michael Marden Claude Poyart}

\title{
Le point sur les transporteurs d'oxygène à base d'hémoglobine
}

Après des années d'espoirs et de déceptions, la recherche d'un transporteur d'oxygène artificiel semble être sortie d'une apparente stagnation. Plusieurs produits ont récemment fait l'objet d'une certaine "publicité »; l'un est un composé de synthèse à base de perfluorocarbure, les autres sont des solutions d'hémoglobine $(\mathrm{Hb})$ humaine modifiée, préparées à partir de sang de banque périmé, ou des $\mathbf{H b}$ recombinantes. Différentes stratégies (pontages chimiques, analogues d'effecteurs, ou mutagenèse dirigée) permettent d'obtenir des solutions d'Hb dont l'affinité pour l'oxygène est similaire à celle des globules rouges humains, et dont la structure tétramérique est stabilisée. La vitesse d'auto-oxydation du fer de l'Hb en solution reste un problème difficile à contrôler. Un obstacle majeur à l'utilisation des transporteurs d'oxygène à base d'H $b$, l'apparition d'une vasoconstriction parfois sévère, sera peut-être franchi grâce à une meilleure connaissance du mécanisme de piégeage du monoxyde d'azote par l'oxyHb.

ADRESSE

L. Pagnier: directeur de recherche à l'Inserm. M. Marden: directeur de recherche à l'Inserm. C. Poyart: directeur de recherche à l'Inserm, Inserm U. 299, Hôpital de Bicêtre, 78, avenue du Général-Leclerc, 94275 Le Kremlin- es média nous ont récemment annoncé l'arrivée prochaine sur le marché de deux produits «substituts du sang" actuellement en cours d'évaluation clinique dans des hôpitaux parisiens: "Bientôt un sang pur artificiel» annonce la page de couverture de Science et Vie [1]; "Le Sang du Futur " titre un hebdomadaire suisse (L'Hebdo, 16 novembre 1995). "Oxygent ${ }^{\circledR}$, produit de synthèse chimique à base de fluorocarbures, est fabriqué dans les laboratoires Alliance Pharmaceutical (La Jolla,
CA, USA), dont un consultant est Jean Riess (Cnrs, URA 426, SophiaAntipolis, France) [2]. L'autre substitut est une solution d'hémoglobine $(\mathrm{Hb})$ d'origine humaine, modifiée chimiquement [3] pour satisfaire aux exigences d'un transporteur d'oxygène (Baxter Healthcare Corp, Round Lake, IL, USA).

Si l'on peut regretter l'emphase journalistique, il n'en reste pas moins que ces deux produits illustrent les progrès spectaculaires effectués depuis une dizaine d'années dans la mise au point d'un 


\section{RÉFÉRENCES}

1. Chambon $P$. Le sang pur sera artificiel Science et Vie 1996 ; 942 : 78-83.

2. Riess JG. Perspectives d'utilisation de transporteurs d'oxygène comme substituts des érythrocytes en chirurgie. Ann Fr Anesth Réanim 1995; 14 : 107-17.

3. Snyder SR, Welty EV, Walder RY, Williams LA, Walder JA. HbXL $99 \mathrm{a}$ : a hemoglobin derivative that is cross-linked between the $\alpha$ subunits is useful as a blood substitute. Proc Natl Acad Sci USA 1987; 84: $7280-4$.

4. Séminaires d'Actualités Transfusionnelles SAT'95, Paris 26 octobre 1995. Transfus Clin Biologique 1995 ; 2 : 423-80.

5. Winslow RM. Hemoglobin-based Red Cell Substitutes. Baltimore and London: The Johns Hopkins University Press, 1992.

6. Bunn HF. The role of hemoglobin based blood substitutes in transfusion medicine. Transfus Clin Biologique 1995; 2: 433-9.

7. Kumar R. Recombinant hemoglobins as blood substitutes: a biotechnology perspective. Proc Soc Exp Biol Med 1995; 208: 150-8.

8. Dietz NM, Joyner MJ, Warner MA. Blood substitutes: fluids, drugs, or miracle solutions? Anesth Analg 1996; 82: 390-405.

9. Zhang L, Levy A, Rifkind JM. Auto-oxydation of hemoglobin enhanced by dissociation into dimers. $J$ Biol Chem 1991; 265: 24698-701.

10. Marden MC, Griffon N, Poyart C. Oxygen delivery and auto-oxydation of hemoglobin. Transfus Clin Biologique 1995; 2 : 47380 .

11. Perutz MF, Fermi G, Poyart C, Pagnier J, Kister J. A novel allosteric mechanism in haemoglobin. Structure of bovine deoxyhaemoglobin, absence of specific chloridebinding sites and origin of chloride-linked Bohr effect in bovine and human haemoglobin. J Mol Biol 1993; 232: 536-45.

$\mathrm{m} / \mathrm{s} n^{\circ} 12$, vol. 12 , décembre 96 transporteur d'oxygène artificiel. Nous allons ici tenter de faire la part entre «le sang du futur» et l'état actuel de la recherche concernant les produits à base d'Hb (HBOC pour hemoglobin based oxygen carrier). Rappelons que la quatrième édition des Séminaires d'Actualité Transfusionnelle (SAT'95), organisés par l'Institut National de la Transfusion Sanguine, a consacré une journée à ce sujet en octobre 1995 [4]. Nous renvoyons le lecteur à un article récent de Riess [2] pour ce qui concerne l'utilisation et les indications cliniques des perfluorocarbones.

Les solutions d'hémoglobine ont depuis longtemps été proposées et testées comme alternative à la transfusion sanguine [5], la source la plus évidente et la plus simple étant l'Hb humaine purifiée à partir de sang de banque périmé. Si cette stratégie permet de s'affranchir de la nécessité du groupage, elle n'élimine pas les risques infectieux contrairement à celle des produits de synthèse chimique comme les perfluorocarbones. Elle restera soumise aux contraintes de l'approvisionnement. En outre, et bien que l'Hb soit le transporteur physiologique de l'oxygène, son utilisation en solution pose certains problèmes liés aux propriétés intrinsèques de la molécule d'Hb hors de son environnement physiologique, tels que toxicité et efficacité fonctionnelle réduite (figure 1) [5-8].

- La toxicité des solutions d'Hb est en relation directe avec la qualité de la purification de la protéine. L'amélioration des techniques autorise maintenant une purification quasi

parfaite, évitant la toxicité rénale liée à la persistance de débris membranaires $[5,6]$. Cependant, même débarrassée des contaminants membranaires, l'Hb en solution diluée reste toxique en raison de la dissociation du tétramère $\alpha_{2} \beta_{2}$ en dimères $\alpha \beta$ (figure 1) filtrés par le rein [6]. La dissociation en dimères a également pour conséquence l'extravasation de l'Hb et une durée de vie très courte dans l'organisme. Elle intervient dans la réduction de l'efficacité du transport de l'oxygène.

- A quels impératifs premiers doit répondre une solution d'Hb pour être utilisable comme transporteur d'oxygène in vivo? (Tableau I): (1) ne présenter ni toxicité, ni risques immunogènes ou infectieux; (2) avoir une capacité de transport de l'oxygène proche de celle du sang; (3) être stable plusieurs mois et, si possible, à température ambiante. La réponse à ces impératifs passe par des changements de structure modifiant certaines propriétés fonctionnelles de l'Hb.

\section{Propriétés fonctionnelles de I'Hb en solution}

Dans les globules rouges, l'affinité de l'Hb humaine pour l'oxygène est modulée par le 2,3-diphosphoglycérate (DPG), effecteur physiologique qui stabilise la structure désoxygénée, de faible affinité pour l'oxygène. Hors du globule rouge, l'absence de DPG a pour conséquence une forte affinité de l'Hb humaine, ce qui limite la capacité de délivrance de l'oxygène aux tissus (figure 1).

\begin{tabular}{|c|}
\hline $\begin{array}{c}\text { Tableau I } \\
\text { LE CAHIER DES CHARGES }\end{array}$ \\
\hline $\begin{array}{l}\text { - produit parfaitement purifié } \\
\text { - non toxique } \\
\text { - non antigénique } \\
\text { - pression oncotique }<25 \mathrm{~mm} \mathrm{Hg} \\
\text { - efficacité du transport de l'oxygène: } \\
\text { affinité pour l'oxygène similaire à celle des globules rouges } \\
\rightarrow \text { oxygénation des tissus } \\
\text { - durée de vie prolongée dans l'organisme } \\
\text { - conservation: } \\
\text { produit stable (idéalement à température ambiante) } \\
\text { faible vitesse d'auto-oxydation }\end{array}$ \\
\hline
\end{tabular}




\begin{tabular}{|c|c|c|c|}
\hline \multicolumn{4}{|c|}{$\begin{array}{l}\text { Tableau II } \\
\text { ORTEURS D'OXYGĖNE À BASE D'Hb } \\
\text { N COURS D'ESSAIS CLINIQUES }\end{array}$} \\
\hline Compagnie & Source d'Hb & Nom & Modification(s) \\
\hline $\begin{array}{l}\text { Baxter Healthcare } \\
\text { Corp. }\end{array}$ & humaine & $\begin{array}{l}\text { HemAssist }{ }^{\mathrm{TM}} \\
\text { (DCLHb) }\end{array}$ & pontage $\alpha-\alpha$ \\
\hline $\begin{array}{l}\text { Northfield Lab. } \\
\text { Inc. }\end{array}$ & humaine & Polyheme $^{\mathrm{TM}}$ & $\begin{array}{l}\text { polymérisation } \\
\text { (glutaraldéhyde) }\end{array}$ \\
\hline Hemosol, Inc. & humaine & Hemolink $^{\mathrm{TM}}$ & $\begin{array}{l}\text { polymérisation } \\
\text { (o-raffinose) }\end{array}$ \\
\hline $\begin{array}{l}\text { Apex Bioscience, } \\
\text { Inc. }\end{array}$ & humaine & & $\begin{array}{l}\text { Hb pyridoxylée } \\
\text { polyoxyéthylène (PHP) }\end{array}$ \\
\hline $\begin{array}{l}\text { BioPure, Corp.; } \\
\text { Upjohn Co }\end{array}$ & bovine & HBOC-201 & polymérisation \\
\hline Enzon Inc. & bovine & & $\begin{array}{l}\text { conjugaison: Hb-PEG } \\
\text { (polyéthylène glycol) }\end{array}$ \\
\hline $\begin{array}{l}\text { Somatogen Inc. } \\
\text { Eli Lilly and Co }\end{array}$ & $\begin{array}{l}\text { Hb humaine } \\
\text { recombinante } \\
\text { (E. coli) }\end{array}$ & $\begin{array}{l}\text { Optro }{ }^{\mathrm{TM}} \\
\text { (rHb1.1) }\end{array}$ & $\begin{array}{l}\text { liaison } \alpha-G l y-\alpha \\
\text { mutation } \beta\end{array}$ \\
\hline
\end{tabular}

L'absence du système enzymatique oxydo-réducteur intracellulaire (metHb réductase) entraîne une autooxydation irréversible avec formation de metHb non fonctionnelle et toxique (figure 1). Les deux phénomènes précédents sont accrus par la dissociation du tétramère en dimères (figure 1). Les dimères $\alpha \beta$ ont une forte affinité pour l'oxygène, et leur vitesse d'auto-oxydation est environ 20 fois supérieure à celle du tétramère d'Hb $[9,10]$.

Par divers moyens, chimiques ou génétiques, on peut diminuer l'affinité de l'Hb pour l'oxygène et/ou stabiliser sa structure tétramérique. Les procédés mis en œuvre sont en partie fonction de l'origine de la solution d'Hb (Tableau II) [2, 3, 5-8, 11-20].

\section{Les principaux substituts à base $d^{\prime} \mathrm{Hb}$ naturelles}

- L'Hb bovine, disponible en grande quantité pour un faible coût, présente l'avantage d'avoir une faible affinité intrinsèque pour l'oxygène, modulée par les ions chlorures, pré- néanmoins nécessaire de stabiliser sa structure tétramérique. L'Hb bovine constitue la matière première des firmes Biopure Corp/Upjohn Co (Boston, MA/ Kalamazoo, MI, USA) et Enzon Inc. (Piscataway, NJ, USA) (Tableau II) [18, 19, 21]. Mais subsistent les risques immunologiques et surtout infectieux: seule l'exclusion formelle de la possibilité de transmission interespèce de l'encéphalite spongiforme, permettrait de développer plus avant un produit à base d'Hb bovine.

- L'Hb humaine purifiée à partir de globules rouges périmés constitue une source beaucoup moins abondante : les dons à risque sont éliminés a priori, et une gestion bien organisée des dons limite le nombre de poches de sang inutilisées. Elle est, certes, plus coûteuse que l'Hb bovine: le criblage effectué dans les centres de transfusion est onéreux mais, à ce prix, les risques viraux sont considérablement réduits. Plusieurs produits sont actuellement en cours d'essais cliniques (Tableau II).

HemAssist $^{\mathrm{TM}}$ : La société Baxter apparaît comme la plus avancée, et l'une des plus solides financièrement, dans le domaine de ces $\mathrm{Hb}$ humaines chimiquement modifiées, avec une préparation d'Hb pontée: diaspirin crosslinked $H b(\mathrm{DCL}-\mathrm{Hb})$ ou HemAssist ${ }^{\mathrm{TM}}$ [22]. Une liaison entre les résidus lysyl en position 99 des deux chaînes $\alpha$, par l'intermédiaire du bis $(3,5-$ dibromosalicyl) fumarate (figure 3) [3], stabilise la structure tétramérique de l'Hb et diminue l'affinité pour l'oxygène. HemAssist ${ }^{\mathrm{TM}}$ est produit depuis quatre ans, selon des procédés industriels contrôlés* incluant la reproductibilité du pontage, l'élimination et l'inactivation des virus par filtration et traitement thermique [22, 23]. L'année 1995 a vu une étape importante franchie pour cette société avec trois séries d'essais cliniques de phase II arrivés à terme aux États-Unis, en Grande-Bretagne et en Belgique: 420 patients ont participé à ces essais, dont 215 traités avec HemAssist ${ }^{\mathrm{TM}}$, les autres recevant une solution saline [22]. Une série de tests a débuté en France, et un protocole d'essais phase III est en cours d'examen par la FDA (Food and Drug Administration)*. Une unité de production est localisée aux ÉtatsUnis, à Round Lake, IL, une autre en Belgique à Lessines; une troisième est en construction en Suisse à Neuchâtel. La commercialisation d'Hem Assist serait prévue pour 1998.

Polyheme, développé par la société américaine Northfield (Evanston, IL, USA) [17] est une Hb humaine polymérisée en présence de glutaraldéhyde (Tableau II). La baisse d'affinité pour l'oxygène est obtenue par ajout de pyridoxal phosphate. C'est (semble-t-il) le produit qui a été testé aux plus fortes doses actuellement: jusqu'à $150 \mathrm{~g}$ chez des malades en état de choc hémorragique [17]. Les responsables de Northfield, comme ceux de Baxter, envisageraient la mise sur le marché de leur substitut pour 1998.

D'autres hémoglobines modifiées ont été testées (Tableau II). Il faut souli-

\footnotetext{
* Good Manufacturing Practice (GMP) facilities: installations et modes de fabrication, contrôles de qualité, etc. répondant aux normes gouvernementales (US Food and Drug Administration ou autres), et concernant la préparation industrielle de produits à usage thérapeutique.
} 


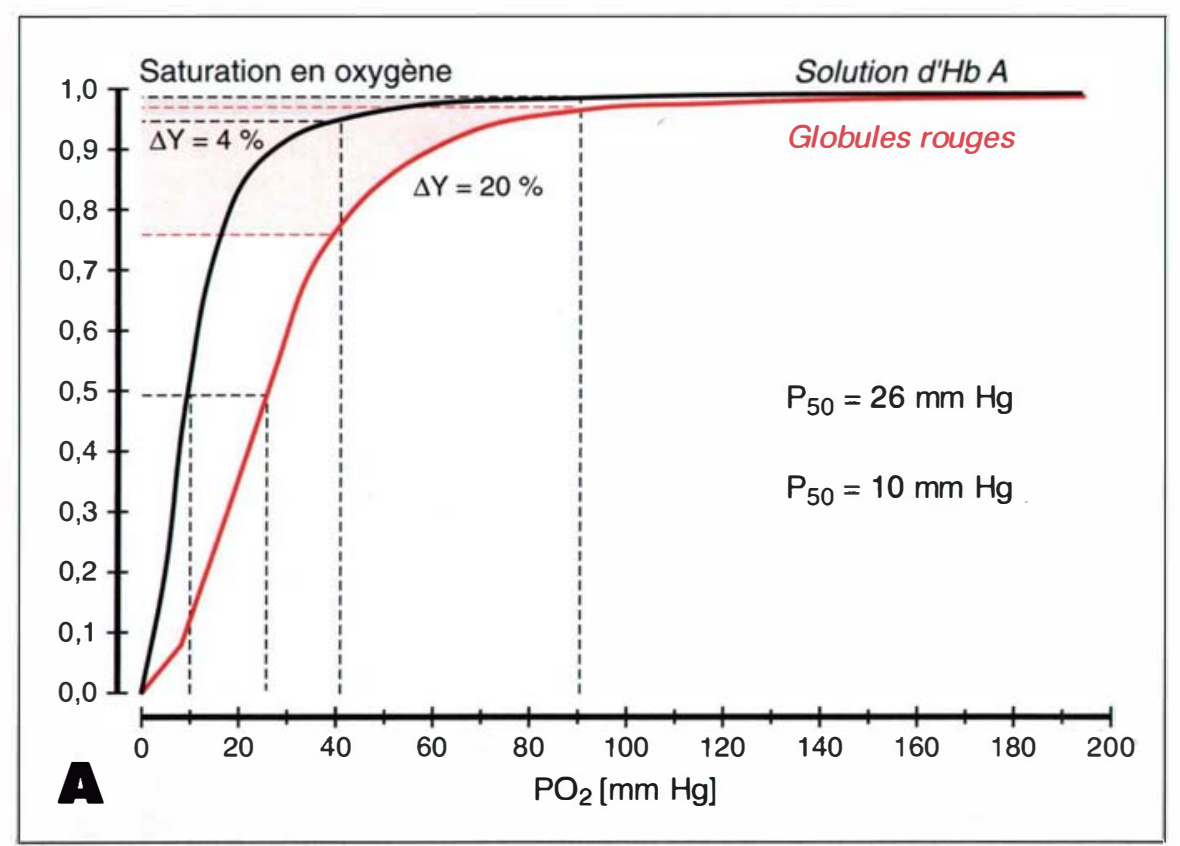

\section{RÉFÉRENCES}

12. Abraham DJ, Kister J, Joshi GS, Marden MC, Poyart C. Intrinsic activity at the molecular level: EJ. Ariëns' concept visualized. $J$ Mol Biol 1995; 248: 845-55.

13. Looker D, Abbott-Brown D, Cozart P, Durfee S, Hoffman S, Mathews AJ, MillerRoehrich J, Shoemaker S, Trimble S, Fermi G, Komiyama NH, Nagai K, Stetler GL. A human recombinant haemoglobin designed for use as a blood substitute. Nature 1992; 356: 258-60.

14. Baudin V, Dumoulin A, Poyart C, Pagnier J. Hémoglobine humaine recombinante de faible affinité pour l'oxygène: effets additionnels de deux mutations. Transfus Clin Biol 1995; 6 : 463-7.

15. Dumoulin A, Kiger L, Griffon N, Vasseur $C$, Kister J, Génin $P$, Marden $M C$, Pagnier J, Poyart C. Two mutations in recombinant $\mathrm{Hb}$ F41 (C7)Y,K82 (EF6)D show additive effects in decreasing the oxygen affinity. Prot Sci 1996; 5: 114-20.

16. Boyiri T, Safo MK, Danso-Danquah RE, Kister J, Poyart C, Abraham DJ. Bisaldehyde allosteric effectors as molecular ratchets and probes. Biochemistry $1995 ; 34$ : 15021-36.

17. Gould SA, Sehgal LR, Sehgal HL, Moss GS. The development of hemoglobin solutions as red cell substitutes. Transfusion Sci $1995 ; 16: 5-17$.

18. Nho K, Zalipsky A, Abuchowski A, Davis FF. PEG-modified hemoglobin as an oxygen carrier. In « Polyethylene Glycol Chemistry: Biotechnical and Biomedical Applications", Plenum, New York; 1992; pp. 171-82.

19. Bradley R, Sloshberg S, Nho K, Czuba B, Szesko D, Shorr R. Production of PEGmodified bovine hemoglobin: economics and feasibility. Art Cells Blood Subs Immob Biotech 1994; 22: 657-67.

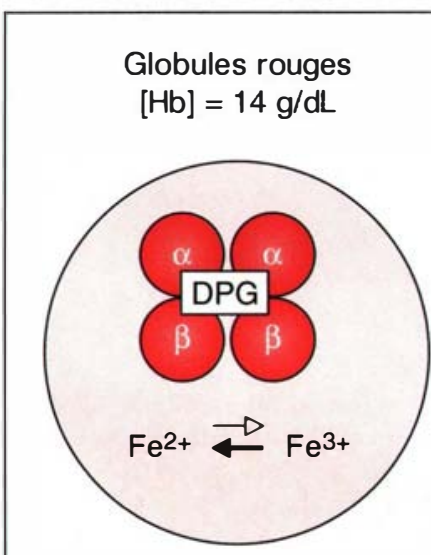

Tétramères $\alpha_{2} \beta_{2}, 64 \mathrm{kDa}$
Solution d'Hb

$[\mathrm{Hb}]=7 \mathrm{~g} / \mathrm{dL}$
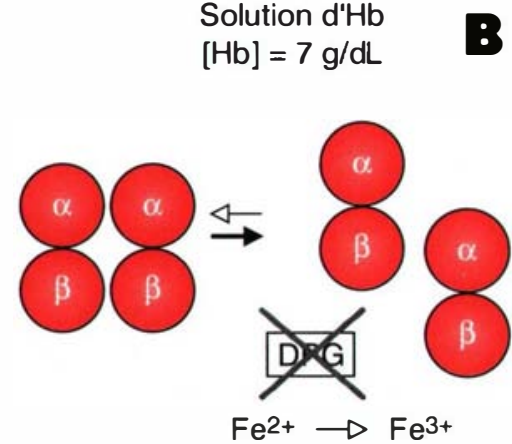

Dimères $\alpha \beta, 32 \mathrm{kDa}$

Figure 1. (A) Courbes de liaison de l'oxygène d'une suspension de globules rouges humains $\left(P_{50}=25-28 \mathrm{~mm} \mathrm{Hg}\right)$ et d'une solution d'Hb humaine purifiée $\left(P_{50}=10-15 \mathrm{~mm} \mathrm{Hg}\right)$, à $37^{\circ} \mathrm{C}, \mathrm{pH} 7,4$ et $140 \mathrm{mM} \mathrm{NaCl}$. Dans les deux cas la courbe est sigmoïde, traduisant le caractère coopératif de la liaison de l'oxygène. Les affinités sont exprimées par la $P_{50}$, pression partielle en oxygène pour laquelle $50 \%$ des molécules $d^{\prime} H b$ sont oxygénées. La différence de $P_{50}$ est due à la présence, dans l'érythrocyte humain, d'un effecteur physiologique, le 2,3-diphosphoglycérate, qui stabilise la forme désoxygénée de l'Hb, de faible affinité pour l'oxygène. Aux valeurs normales de la pression partielle en oxygène $\left(\mathrm{PO}_{2}\right)$ alvéolaire, les globules rouges et la solution d'Hb sont saturées en $\mathrm{O}_{2}$ à $98 \%$. La délivrance $d^{\prime} \mathrm{O}_{2}$ au niveau des tissus $\left(\mathrm{PO}_{2}=\right.$ $40 \mathrm{~mm} \mathrm{Hg}$ ), représente $20 \%$ de l'O $\mathrm{O}_{2}$ fixé pour les globules rouges, contre seulement $4 \%$ pour l'Hb en solution $(\Delta Y=$ différence de saturation de l'Hb par $\left.l^{\prime} \mathrm{O}_{2}\right)$. Hors du globule rouge, l'affinité de $\mathrm{I}^{\prime} \mathrm{Hb}$ pour $\mathrm{l}^{\prime} \mathrm{O}_{2}$ est donc trop élevée pour permettre l'oxygénation adéquate des tissus. Ce phénomène est accentué par la dissociation du tétramère $d^{\prime} \mathrm{Hb} \alpha_{2} \beta_{2}$ en dimères $\alpha \beta$ (B), de forte affinité pour l'oxygène. Avec une solution injectable de même concentration en $\mathrm{Hb}$ que dans le globule rouge, la pression oncotique serait trop élevée. La dissociation en dimères favorise: (1) l'élimination rapide par voie rénale, d'où durée de vie courte et néphrotoxicité; (2) le passage de la barrière endothéliale et la diffusion de l'Hb dans les tissus d'où le piégeage de l'oxyde d'azote et la vasoconstriction consécutive. 


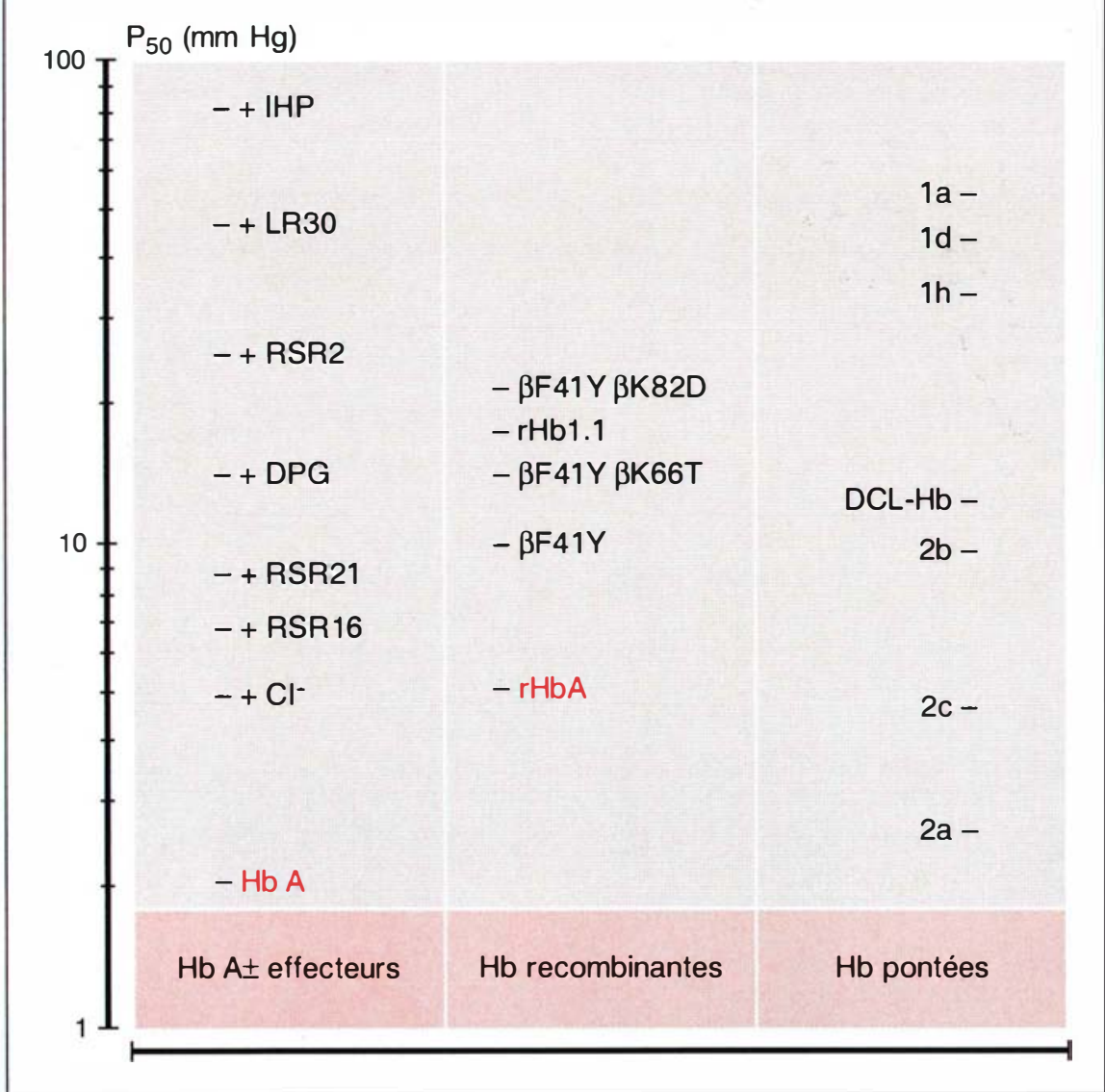

Figure 2. Affinité pour l'oxygène de solutions d'Hb: (1) Hb humaine (Hb A) purifiée, en absence et en présence de divers effecteurs physiologiques (DPG, $\mathrm{Cl}^{-}$) [11] ou synthétiques (RSR et LR 30: dérivés du bézafibrate; IHP: inositol hexaphosphate) [12]; (2) Hb humaines recombinantes: $r H b A=$ normale; rHb1.1 = tétramère stabilisé par une liaison covalente (lien peptidique) entre les extrémités $N$ - et $C$-terminales des deux sous-unités $\alpha$ (Somatogen); $\mathrm{Hb}$ recombinantes mutées [13-15]; (3) Hb humaines pontées chimiquement entre la valine $N$-terminale d'une chaîne $\alpha$ et la lysine 99 de l'autre chaîne $\alpha$ : $D C L-H b=$ diaspirin cross-linked [3, 22]; les autres $H b$ résultent elles aussi d'un pontage mais par réaction avec des composés aldéhydiques. Ces $\mathrm{Hb}$ diffèrent par la longueur de la chaîne carbonée unissant deux dimères: 1a = 1 groupe $\mathrm{CH}_{2}, 1 d=4$ groupes $\mathrm{CH}_{2}, 1 \mathrm{~h}=10$ groupes $\mathrm{CH}_{2}$, et par la position en méta (2a), ortho (2b) ou para (2c) des groupes $\mathrm{CH}_{2}$ en milieu de chaîne [16]. Les affinités pour I'oxygène des $\mathrm{Hb}$ recombinantes $\beta F 41 Y, K 82 D, \beta F 41 Y$, $K 66 T$, de la rHb1.1 et de la DCL-Hb sont proches de celle de l'Hb A en présence de DPG. Elles ont été déterminées à $25^{\circ} \mathrm{C}, \mathrm{pH} 7,4$ et en présence de $\mathrm{NaCl} 100 \mathrm{mM}$ (à l'exception du témoin $\mathrm{Hb}$ A).

gner qu'il est extrêmement difficile d'obtenir des informations précises. En particulier, les résultats d'essais cliniques restent partiels et trop longtemps confidentiels, car mettant en jeu des intérêts financiers importants. Lors de la Conférence "Blood Substitutes and Related Products» qui
1995, les conclusions des essais cliniques (phase II) effectués par différentes firmes (Baxter, Northfield, Somatogen...) pouvaient être résumés en une ligne: résultats encourageants, pas d'effets nocifs imputables à l'injection du produit [24]. Ajoutons qu'il est difficile d'évaluer l'efficacité de ces solutions.

\section{Les hémoglobines recombinantes}

L'ingénierie des protéines, approche plus récente (une dizaine d'années) a connu un essor particulier. Les progrès ont été rapides et remarquables puisqu'un produit issu de ces technologies [13], mis au point par la société Somatogen Inc. (Boulder, CO, USA) récemment associée aux laboratoires Eli Lilly and Co (Indianapolis, IN, USA), est en phase d'essais précliniques [25].

\section{Systèmes d'expression}

Plusieurs systèmes d'expression sont maintenant éprouvés pour obtenir de l'Hb recombinante, avec la possibilité de modifier génétiquement la molécule d'Hb [7]. La co-expression des sous-unités d'Hb dans Escherichia coli [26] ou Saccharomyces cerevisiae [27], avec accumulation d'Hb soluble dans le cytoplasme de la cellule productrice, présente l'avantage, au moins a priori, d'éliminer les risques infectieux. Des porcs transgéniques produisent également de l' $\mathrm{Hb}$ humaine [28]; mais on retrouve alors les possibilités de transmissions virales chez l'homme. Que l'hôte choisi pour l'expression soit un micro-organisme ou le porc, les procédés de purification sont complexes, puisqu'il faut éliminer les contaminants d'origine bactérienne ou animale présents en quantité importante. D'autres problèmes sont loin d'être négligeables, tel l'aspect religieux lié à l'utilisation d'un composé issu du porc.

\section{Ingénierie des protéines}

En contrepartie, l'ingénierie des protéines permet de produire des hémoglobines mutées dont les propriétés fonctionnelles sont modifiées [7, 1315, 29-32]. Il n'y a donc pas lieu de les traiter ensuite chimiquement. Plusieurs stratégies visant à diminuer l'affinité de l'Hb recombinante ont été décrites. Elles s'appuient à la fois sur les données cristallographiques et la modélisation moléculaire de l'Hb [33], et la connaissance des propriétés d'autres $\mathrm{Hb}$ animales [11, 29-31] et de mutants naturels de l'Hb humaine [13-15]. Les modifications " idéales" ne doivent entraîner que 


\section{RÉFÉRENCES}

20. Menu P, Faivre B, Labrude P, Riffard P, Grandgeorge M, Vigneron C. Human hemoglobin conjugated to carboxylate dextran as a potential red blood cell substitute. II. Pharmacotoxicological evaluation. Art Cells Blood Subs Immob Biotech 1994; 22 : 5439.

21. Hughes GS, Yancey EP, Albrecht R, Locker P, Francom SF, Orringer EP, Antal EJ, Jacobs EE. Hemoglobin-based oxygen carrier preserves submaximal exercise capacity in humans. Clin Pharmacol Ther 1995; 58: 434-43.

22. Estep TN. Recent progress in the development of DCLHb ${ }^{\mathrm{TM}}$ solutions as a hemoglobin-based therapeutic. Third Annual Conference on "Blood Substitutes and Related Products». October 30-31, 1995, Bethesda, MD. Program and Abstracts.

23. Farmer $M$, Ebeling A, Marshall $T$, Hauck W, Sun CS, White E, Long Z. Validation of virus inactivation by heat treatment in the manufacture of diaspirin crosslinked hemoglobin. Biomat Art Cells Immob Biotech $1992 ; 20: 429-33$.

24. Third Annual Conference on «Blood Substitutes and Related Products". October 30-31, 1995, Bethesda, MD. Program and Abstracts.

25. Caspari RF. Early phase II clinical experience with Optro ${ }^{\mathrm{TM}}$ recombinant human hemoglobin. Third Annual Conference on "Blood Substitutes and Related Products". October 30-31, 1995, Bethesda, MD. Program and Abstracts.

26. Hoffman SJ, Looker DL, Roehrich JM, Cozart PE, Durfee SL, Tedesco JL, Stetler GL. Expression of fully functional tetrameric human hemoglobin in Escherichia coli. Proc Natl Acad Sci USA 1990; 87: 8521-5.

27. Wagenbach $\mathbf{M}, O^{\prime}$ Rourke $K$, Vitez $\mathbf{L}$, Wieczorek A, Hoffman S, Durfee S, Tedesco J, Stetler G. Synthesis of wild type and mutant human hemoglobins in Saccharomyces cerevisiae. Biotechnol 1991; 9 : 57-61.

28. Swanson ME, Martin MJ, O'Donnell JK, Hoover K, Lago W, Huntress V, Parsons CT, Pinkert CA, Pilder S, Logan JS. Production of functional human hemoglobin in transgenic swine. Biotechnol 1992; 10, 557-9.

29. Baudin V, Bihoreau MT, Kister J, Marden M, Pagnier J, Poyart C. Functional properties of $\boldsymbol{\beta}$ (NA1) Val-deleted, (NA2)His $\rightarrow$ Met hemoglobin synthesized in Escherichia coli. Art Cells Blood Subs Immob Biotechnol 1994 ; 22 : 739-45.

$\mathrm{m} / \mathrm{s} n^{\circ} 12$, vol. 12 , décembre 96

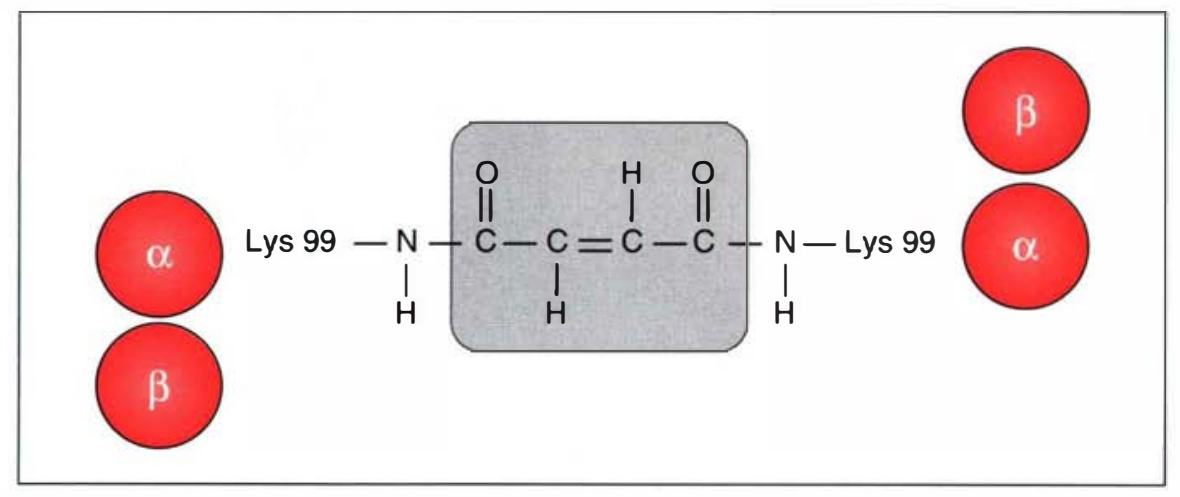

Figure 3. Pontage intramoléculaire (fond gris) de l'Hb humaine entre les résidus lysyl (G6) 99 des chaînes $\alpha$ obtenu par réaction avec un agent bifonctionnel, le bis 3,5-dibromosalicyl fumarate. Si la réaction de pontage est effectuée en anaérobiose, l'Hb pontée a une affinité réduite pour l'oxygène. Elle présente également une stabilité thermique augmentée comparée à l'Hb non pontée, ce qui permet l'inactivation virale par traitement thermique [23].

des modifications structurales discrètes, et une affinité pour l'oxygène similaire à celle de l'Hb dans les globules rouges, soit 25 à $30 \mathrm{~mm} \mathrm{Hg}$ à $37^{\circ} \mathrm{C}, \mathrm{pH} 7,4$ (figure 1).

Une approche intéressante, utilisée par Baudin et al. [14], consiste à associer des mutations abaissant l'affinité de l'Hb pour l'oxygène par des mécanimes différents: en fonction des positions relatives des sites de mutations, les deux mécanismes sont susceptibles d'agir indépendamment, et l'on observe alors une additivité des effets. Les résultats montrent qu'en introduisant des mutations convenablement choisies, on peut moduler la capacité de transport de l'oxygène par les solutions d'Hb [14, 15].

Le groupe de Poyart en France, celui de Fronticelli aux États-Unis, ont utilisé le modèle que représente l'Hb bovine afin de transplanter, dans l'Hb humaine, les résidus responsables de la faible affinité intrinsèque pour l'oxygène de l'Hb bovine en l'absence de DPG [11, 29-31]. Nagai et al. ont également utilisé l'ingénierie des protéines dans le but de mimer les propriétés de l'Hb de crocodile [32]. Dans ces deux cas, l'introduction de multiples mutations a infléchi les propriétés de l'Hb humaine dans le sens d'une diminution de l'affinité pour l'oxygène, mais encore insuffisamment pour autoriser une application thérapeutique. L'Hb développée par Somatogen, rHbl.1 [7, 13] ou Optro ${ }^{\mathrm{TM}}$, est produite dans $E$. coli sous la forme d'un tétramère stabilisé par une liaison covalente (lien peptidique) entre les extrémités $\mathrm{N}$ - et C-terminales des deux sous-unités $\alpha$. La baisse d'affinité pour l'oxygène est obtenue par introduction d'une mutation naturelle de la chaîne $\beta$ ( $\beta^{108}$ Asn $\rightarrow$ Lys). Ces exemples montrent qu'il est possible de modifier l'Hb, chimiquement et génétiquement, afin d'abaisser l'affinité pour l'oxygène et stabiliser la structure tétramérique. Ces deux conditions sont nécessaires mais pas suffisantes; certains problèmes subsistent: (1) l'Hb en solution s'auto-oxyde rapidement en metHb non fonctionnelle $[9,10]$; (2) un effet vasoconstricteur des solutions d'Hb est observé de façon quasi constante lors des essais cliniques [34, 35]; (3) l'Hb et ses produits de dégradation, hème, atomes de fer libres, pourraient favoriser la croissance bactérienne, rendant le receveur particulièrement sensible aux infections graves pouvant évoluer jusqu'à la septicémie [36]. La liaison de l'Hb à l'endotoxine bactérienne (lipopolysaccharide) serait responsable de certains de ces effets toxiques des solutions d'Hb [37]. 


\section{Problèmes inhérents à I'Hb en solution}

Plusieurs groupes de recherche étudient les effets délétères de l'oxydation de l'hème sur les propriétés fonctionnelles de l'Hb. Le mécanisme est complexe et encore mal connu [10]. Le fer héminique des molécules d'Hb en solution s'oxyde spontanément au contact de l'oxygène: le taux d'oxydation est d'environ $50 \%$ en deux jours à $37{ }^{\circ} \mathrm{C}$, ce qui signifie que plus de la moitié des molécules d'Hb contiennent du fer ferrique, forme impropre au transport de l'oxygène. Dans les globules rouges, qui circulent environ 120 jours, la "réactivation " de l'Hb est assurée par l'activité d'un sytème d'oxydo-réduction enzymatique (metHb réductase). L'ajout d'un tel système enzymatique renouvelable dans les solutions d'Hb purifiée n'est pas encore envisageable. En absence de réductase l'oxydation est irréversible, et le temps de vie d'un transporteur d'oxygène à base d'Hb sera limité. Le contrôle simultané des différents paramètres, affinité pour l'oxygène, équilibre dimères-tétramères vitesse d'auto-oxydation, représente un défi pour les chercheurs, car ces paramètres ne sont pas indépendants. La dimérisation et l'oxydation partielle entraînent une augmentation de l'affinité pour l'oxygène; la dimérisation et la diminution de l'affinité pour l'oxygène accélèrent la vitesse d'auto-oxydation. Limiter la vitesse d'auto-oxydation est d'autant plus difficile que, en règle générale, cette vitesse augmente d'autant plus que baisse l'affinité pour l'oxygène. Il a été montré que l'oxydation d'une seule sous-unité d'un tétramère d'Hb modifie le comportement fonctionnel de l'ensemble de la molécule en déplaçant la courbe d'oxygénation vers les fortes affinités. La capacité de délivrance de l'oxygène est alors diminuée [10]. Plutôt que de chercher à découpler ces effets, il semble actuellement possible de trouver un compromis acceptable entre les différents effets.

Les diverses mutations réalisées n'ont malheureusement pas conduit à une diminution des phénomènes d'autooxydation. Nous disposons d'Hb recombinantes de faible affinité pour l'oxygène qui, en l'absence de DPG nelles similaires à celles de l'Hb humaine native en présence de DPG. La vitesse d'auto-oxydation de l'une de ces $\mathrm{Hb}$ mutées reste similaire à celle de l'Hb humaine, malgré la diminution de l'affinité. Les possibilités de pontage ou d'encapsulation laissent prévoir des améliorations. D'autre progrès pour améliorer les propriétés des $\mathrm{Hb}$ recombinantes ou chimiquement modifiées, dans l'optique d'une utilisation thérapeutique, ne pourront être réalisés qu'avec une connaissance très précise des divers facteurs impliqués dans la stabilité et la régulation de l'affinité vis-à-vis des ligands. Ces facteurs, notamment la nature et le rôle des acides aminés essentiels, nécessaires à l'obtention d'une molécule d'Hb stable, fixant et libérant l'oxygène de façon réversible seront de mieux en mieux maîtrisés.

Les limites des possibilités d'action directe sur la molécule d'Hb ont eu pour conséquence le développement des recherches sur des procédés de conservation protégeant l'hème de l'oxydation dans les solutions d'Hb.

\section{Conservation des HBOC}

Certains sucres, comme le tréhalose, pourraient constituer un milieu de conservation des HBOC. Ce disaccharide naturel (figure 4) s'accumule à forte concentration dans de nombreux organismes soumis à des conditions extrêmes de température et de dessiccation. Il aurait un rôle dans la préservation des structures biologiques [38]. Il existe sous plusieurs formes solides, dont une forme amorphe vitreuse, milieu très visqueux dans lequel la diffusion des réactifs impliqués dans une réaction bimoléculaire serait ralentie, d'où des vitesses de réaction plus faibles. Le tréhalose posséderait les propriétés nécessaires pour la préservation des solutions d'Hb [39]: absence de toxicité, solubilisation de l'Hb à concentration élevée, ralentissement de la vitesse d'oxydation donc de la formation de metHb, stabilité à température ambiante facilitant le stockage et le transport, préparation aisée et rapide. Il pourrait également être utilisé dans la perspective de l'encapsulation de l'Hb, avec un double effet: protection de l'Hb contre l'oxydation et stabilisation $\mathrm{du}$ vecteur d'encapsulation. Cette hypo- thèse intéressante est actuellement en cours d'étude [39].

La vectorisation de l'Hb permettrait également de pallier plusieurs des inconvénients des HBOC. Elle autoriserait l'ajout de co-facteurs et/ou de "conservateurs", et éviterait peutêtre d'avoir à modifier la molécule d'Hb. L'approche de Samain et al. [40] consiste à construire des vecteurs synthétiques biomimétiques des érythrocytes, caractérisés par la présence d'une membrane phospholipidique autour d'un cytosquelette. Des résultats préliminaires montrent qu'il est possible d'atteindre des taux de fixation élevés d'Hb sur des matrices polysaccharidiques jouant le rôle de cytosquelette.

Après la mise en évidence du rôle du monoxyde d'azote (NO), agent vasodilatateur et élément régulateur du tonus des fibres vasculaires lisses, l'effet vasoconstricteur des solutions

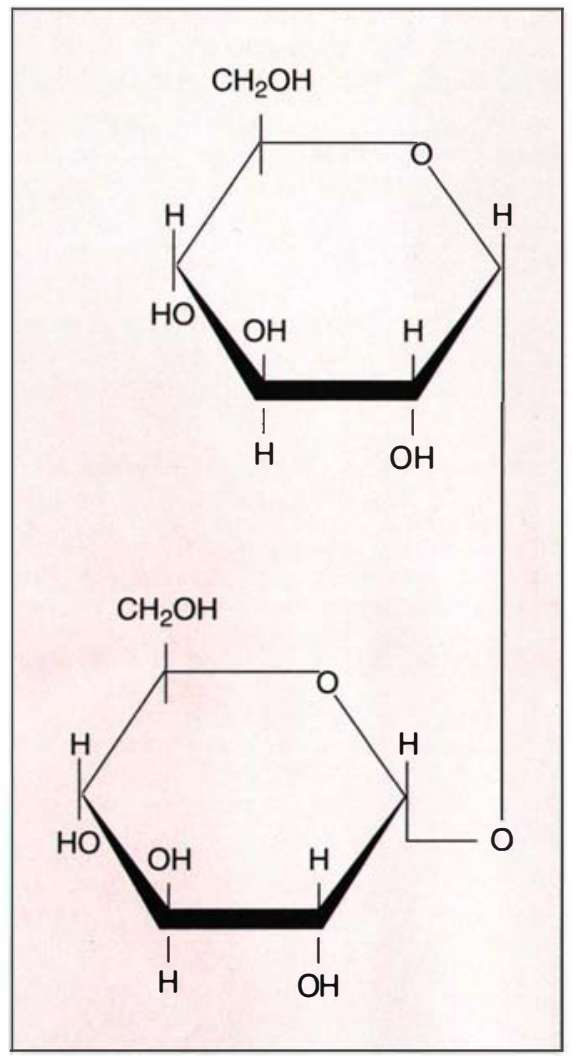

Figure 4. Le tréhalose pourrait constituer un milieu de conservation pour les solutions d'Hb. C'est un sucre naturel non réducteur et biologiquement inerte, clivé dans le rein en deux molécules de glucose par une disaccharidase. 


\section{RÉFÉRENCES}

30. Fronticelli C, Sanna MT, Perez-Alvarado GC, Karavitis M, Lu AL, Brinigar WS. Allosteric modulation by tertiary structure in mammalian hemoglobins. J Biol Chem 1995; 270: 30588-92.

31. Baudin V, Kister J, Poyart C, Pagnier J. Transfert des propriétés fonctionnelles de l'hémoglobine bovine à l'hémoglobine humaine. Transfus Clin Biologique 1995; 6: 469-71.

32. Komiyama $\mathrm{NH}$, Miyazaki G, Tame J, Nagai K. Transplanting a unique allosteric effect from crocodile into human haemoglobin. Nature 1995; 273: 2446.

33. Fermi G, Perutz MF, Shaanan B, Fourme R. The crystal structure of human deoxyhaemoglobin at $1.74 \AA$ resolution. $J$ Mol Biol 1984; 175 : 159-74.

34. Alayash AI, Fratantoni JC, Bonaventura C, Bonaventura J, Cashon RE. Nitric oxide binding to human ferrihemoglobins crosslinked between either $\alpha$ or $\beta$ subunits. Arch Biochem Biophys 1993; 303: 332-8.

35. Rioux F, Petitclerc E, Audet R, Drapeau G, Fielding RM, Marceau F. Recombinant human hemoglobin inhibits both constitutive and cytokine-induced nitric oxidemediated relaxation of rabbit isolated aortic rings. J Cardiovasc Pharmacol 1994; 24 : 22937.

36. Griffiths E, Cortes A, Gilbert N, Stevenson P, MacDonald S, Pepper D. Haemoglobin-based blood substitutes and sepsis. Lancet $1995 ; 345: 158-9$.

37. Kaca W, Roth RI, Levin J. Hemoglobin, a newly recognized lipopolysaccharide (LPS)-binding protein that enhances LPS biological activity. J Biol Chem 1994; 269: 25078-84.

38. Newman YM, Ring SG, Colaco C. The role of trehalose and other carbohydrates in biopreservation. Biotechnol Genet Engineering Rev 1993; 11 : 263-94.

39. Hagen SJ, Hofrichter HJ, Bunn HF, Eaton WA. Comments on the physics and chemistry of trehalose as a storage medium for hemoglobin-based blood substitutes. Transfus Clin Biologique 1995; 2 : 423-6.

40. Samain D, Leclerc L, Bonnafoux M, Moulai-Ali S, Kister J, Poyart C. Conception et développement d'une nouvelle génération de transporteur d'oxygène. Transfus Clin Biologique 1995; 2 : 449-52.

41. Jia L, Bonaventura C, Bonaventura J, Stamler JS. S-nitrosohaemoglobin: a dynamic activity of blood involved in vascular control. Nature 1996; 380 : 221-6.

42. Dieryck W, Gruber V, Baudino S, Lenée P, Pagnier J, Mérot B, Poyart C. Expression d'hémoglobine humaine recombinante dans les plantes. Transfus Clin Biologique $1995 ; 2$ : 441-7. d' $\mathrm{HbO}_{2}$, a été attribué à la fixation du monoxyde d'azote par l'Hb. L'interaction $\mathrm{Hb}-\mathrm{NO}$ surviendrait en raison de la fuite de l'Hb en solution dans l'espace extravasculaire, quand le NO diffuse à partir des cellules endothéliales vers les fibres musculaires lisses [34, 35]. Une hypothèse plus récente attribue aux thiols (cystéine, glutathion, albumine du plasma) un rôle de protection du NO en présence d' $\mathrm{HbO}_{2}$, permettant de maintenir sa fonction vasodilatatrice (m/s $n^{\circ}$ 6-7, vol. 12, p. 848) [41].

\section{Développement d'un système $d^{\prime}$ expression alternatif pour I'Hb recombinante}

Plusieurs systèmes de production ont été testés. L'Hb recombinante proposée par Somatogen est synthétisée dans E. coli [13, 25, 26], alors que l'organisme le plus apte à une production de masse serait le porc [28]. Est-il possible de produire des quantités suffisantes d'Hb par fermentation dans $E$. coli? Qu'en sera-t-il de l'élimination des déchets? Les problèmes de purification sont-ils entièrement résolus? Ces questions sont également importantes en ce qui concerne la séparation des différentes $\mathrm{Hb}$ à partir des porcs transgéniques, et quelles seraient les nuisances autour d'une unité géante d'élevage de porcs?

Depuis plus de cinq ans plusieurs auteurs ont décrit la synthèse de protéines d'intérêt thérapeutique par des plantes transgéniques [42]. A notre connaissance, rien n'a été décrit concernant l'expression d'Hb par ces organismes, dont l'intérêt potentiel est à considérer: les plantes apporteraient une sécurité maximale vis-à-vis des agents pathogènes animaux; la culture en champs de plantes transgéniques permettrait la production en masse et à faible coût de protéines recombinantes. Des essais sont en cours pour l'obtention de plantes transgéniques produisant de l'Hb humaine recombinante fonctionnelle [42].

\section{Conclusion}

Grâce aux efforts de recherche théorique et appliquée, les progrès réalisés ces dernières années ont été considérables. Il semble acquis que des transporteurs d'oxygène seront utilisés dans un avenir relativement proche. Ces produits de première génération ne visent en aucun cas à concurrencer la transfusion sanguine "traditionnelle». Ils apparaissent comme une alternative ou un additif dans des indications limitées, en particulier en chirurgie et médecine d'urgence: une rétention intravasculaire de 24 heures serait peut-être suffisante pour éviter les riques d'hypoxie aiguë et représenterait un avantage considérable. Ils auraient également une utilité certaine dans la préservation d'organes. A plus long terme, et pour pouvoir étendre le champ d'application, il serait souhaitable de mettre au point un procédé de production répondant aux besoins quantitatifs, et cela à un coût raisonnable

\section{Remerciements}

Ce travail a été effectué dans l'Unité Inserm 299. Il a été financé par l'Inserm, la Direction de la Recherche et de la Technologie (contrat 92/177), Air Liquide Co et la Faculté de Médecine Paris-Sud.

TIRÉS À PART

J. Pagnier.

\section{Note ajoutée aux épreuves}

En septembre 1996, la société Baxter s'est implantée en France : un accord de partenariat a été conclu avec LFB (Laboratoire français du fractionnement) qui produira à Lille la Diaspirin cross-linked hemoglobin. Rappelons que le LFB est un groupement d'intérêt public sans but lucratif, créé en 1994 après la réforme de la Transfusion sanguine. 


\section{Summary}

Current status of hemoglobinbased blood substitutes

After years of hope and frustation, research on artificial oxygen carriers seems to have exited from an apparent stagnation. Several products have recently been the subject of a certain publicity: one is a synthetic compound based on perfluorocarbons, while others are solutions of modified human hemoglobin ( $\mathrm{Hb})$. These latter transporters are prepared from outdated bank blood or from recombinant $\mathrm{Hb}$. Much progress have been made in controlling the oxygen affinity of $\mathrm{Hb}$ solutions. Several strategies, based on mutants or cross-links, have lead to $\mathrm{Hb}$ solutions with properties similar to those of natural erythrocytes. The lack of the physiological effector 2.3-diphosphoglycerate in the purified $\mathrm{Hb}$ solutions can be compensated for by a combination of mutations, or by the type of crosslinking. Unfortunately, less progress have been made to compensate the loss of the reducing system, and the rate of oxidation may limit the useful lifetime of these solutions. A major obstacle in the utilisation of the $\mathrm{Hb}$ based oxygen carriers ( $\mathrm{HBOC}$ ) is the appearance of a vasoconstriction, which may be overcome through a better understanding of the mechanism of interaction of $\mathrm{NO}$ with $\mathrm{Hb}$. Apart from this effect, the initial clinical tests have not revealed a toxicity to the exposure of $\mathrm{Hb}$ solutions. There is thus some optimism that the functional properties can be improved to produce a useful HBOC.

\section{APPEL D'OFFRES}

PRIX

\section{INSERM/ACADEMIE DES SCIENCES \\ 1997}

Un prix d'un montant total de $200000 \mathrm{FF}$, destiné à apporter un soutien important aux travaux de recherche d'une équipe signataire

d'une Note de grande portée scientifique publiée

dans les Comptes Rendus de l'Académie des Sciences, dans les domaines de la biologie fondamentale, des sciences biomédicales et de la santé, sera attribué en 1997.

Les notes concourant pour ce Prix auront été publiées dans les Comptes Rendus de l'Académie des Sciences des années 1994, 1995, 1996, Série III (Sciences de la Vie).

Elles doivent faire l'objet d'une proposition par une personnalité scientifique ou par les auteurs eux-mêmes.

L'évaluation des dossiers sera effectuée

par le Conseil scientifique del'INSERM et l'Académie des Sciences.

Les dossiers de candidature, disponibles à l'INSERM et au secrétariat de l'Académie des Sciences, doivent être adressés à l'INSERM.

Date limite de dépôt des candidatures : 28 mars 1997.

La décision d'attribution du Prix sera communiquée fin juillet 1997.
Institut National de la Santé et de la Recherche Médicale Département de l'Information et de la Communication 101, rue de Tolbiac 75654 Paris cedex 13

Tél. : 01.44.23.60.71
Académie des Sciences Secrétariat

23, quai de Conti - 75006 Paris

Tél. : 01.44.41.43.83

\section{ACADEMIE DES SCIENCES}

\section{INSTITUT NATIONAL DE LA SANTE ET DE LA RECHERCHE MEDICALE}

